\title{
Análisis de aguas superficiales con alto contenido de fosfatos para el diseño de una planta de tratamiento de
} agua potable

\author{
Analysis of surface waters with high phosphate content for the design of a \\ drinking water treatment plant
}

Ana Gabriela Flores Huilcapi. ${ }^{1}$, Luis Santiago Carrera Almendáriz. ${ }^{2}$ \& Carlos Alcíbar Medina Serrano. ${ }^{3}$

Recibido: 11-04-2020 / Revisado: 16-05-2020 /Aceptado: 08-06-2020 / Publicado: 03-07-2020

\section{Resumen.}

DOI: https://doi.org/10.33262/concienciadigital.v3i3.1264

El objetivo de esta investigación es el diseño de una planta de tratamiento de agua potable a partir de aguas superficiales. El muestreo del agua cruda de la captación se realiza sistemáticamente durante cuatro semanas consecutivas; las muestras tomadas fueron caracterizadas en un laboratorio de control de calidad mediante pruebas físico-químicas y microbiológica según la Norma Técnica INEN 1108:2014 referida a Requisitos de Agua Potable. Se identifica que las muestras de agua contienen concentraciones de fosfatos y turbidez fuera de los límites permisibles según normativa vigente, para turbiedad 5 NTU y para fosfatos 0,1 $\mathrm{mg} / \mathrm{L}$. Para disminuir la concentración de fosfatos de desarrollan pruebas de tratabilidad a nivel de laboratorio, realizando dosificaciones de sulfato de cobre en solución por cada litro de agua cruda. La adición de $5 \mathrm{mg} / \mathrm{L}$ de sulfato de cobre disminuye la concentración de fosfatos en $82,5 \%$, parámetro que está dentro de la norma establecida. A partir de un caudal máximo de tratamiento de $24 \mathrm{~L} / \mathrm{s}$ de captación de agua superficial se realizan cálculos de ingeniería y diseño para la planta de tratamiento de agua potable que contiene un medidor de caudales, dos sedimentadores, dos filtros ascendentes gruesos y tres filtros lentos con arena fina.

\footnotetext{
${ }^{1}$ Escuela Superior Politécnica de Chimborazo, Facultad de Mecánica, Riobamba, Ecuador, ana.flores@espoch.edu.ec, ORCID: 0000-0001-8748-7859

${ }^{2}$ Escuela Superior Politécnica de Chimborazo, Facultad de Ciencias, Riobamba, Ecuador, luissantiago.carrera@espoch.edu.ec, ORCID: 0000-0002-3262-5895

${ }^{3}$ Escuela Superior Politécnica de Chimborazo, Facultad de Ciencias, Riobamba, Ecuador, carlos.medinas@espoch.edu.ec, ORCID: 0000-0003-4916-72421
} 
El medidor de caudales es tipo parshall con un ancho de garganta de $\mathrm{W}=0,229$; el sedimentador clásico con una velocidad de sedimentación crítica Vsc $=0,26$ $\mathrm{mm} / \mathrm{s}$; el filtro ascendente grueso con una velocidad de filtración de $\mathrm{Vf}=0,6 \mathrm{~m} / \mathrm{h}$ y el filtro lento con una velocidad de filtración de $\mathrm{Vf}=0,3 \mathrm{~m} / \mathrm{h}$.

Palabras clave: Agua potable, Fosfatos, Sedimentador, Filtro, Drinking water, Phosphates, Settler, Filter

\begin{abstract}
.
The objective of this research is the design of a drinking water treatment plant from surface waters. The sampling of the raw water from the catchment is carried out systematically for four consecutive weeks; The samples taken were characterized in a quality control laboratory through physical-chemical and microbiological tests according to Technical Standard INEN 1108: 2014 referring to Drinking Water Requirements. It is identified that the water samples contain phosphate concentrations and turbidity outside the permissible limits according to current regulations, for turbidity $5 \mathrm{NTU}$ and for phosphates $0,1 \mathrm{mg} / \mathrm{L}$. In order to reduce the phosphate concentration, laboratory-level treatability tests are carried out, carrying out dosages of copper sulfate in solution for each liter of raw water. The addition of $5 \mathrm{mg} / \mathrm{L}$ of copper sulfate decreases the phosphate concentration by $82.5 \%$, a parameter that is within the established norm. The addition of $5 \mathrm{mg} /$ L of copper sulfate decreases the phosphate concentration by $82,5 \%$, a parameter that is within the established norm. Based on a maximum treatment flow rate of $24 \mathrm{~L} / \mathrm{s}$ of surface water collection, engineering and design calculations are carried out for the drinking water treatment plant that contains a flow meter, two settlers, two thick rising filters and three slow filters. with fine sand. The flow meter is a parshall type with a throat width of $\mathrm{W}=0,229$; the classic settler with a critical settling speed $\mathrm{Vsc}=0,26 \mathrm{~mm} / \mathrm{s}$; the thick rising filter with a filtration speed of $\mathrm{Vf}=0,6 \mathrm{~m} / \mathrm{h}$ and the slow filter with a filtration speed of $\mathrm{Vf}=0,3 \mathrm{~m} / \mathrm{h}$.
\end{abstract}

Keywords: Drinking water, Phosphates, Sedimentator, Filter, Drinking water, Phosphates, Settler, Filter

\title{
Introducción.
}

El agua, es el recurso más abundante en el planeta y la base de la vida. En nuestro planeta el agua ocupa una alta proporción en relación con la zona continental alrededor de un 71\%. (Guerrero, 1976). El agua superficial está formada de escurrimientos directos y del flujo "básico", es decir, aquella porción de la descarga de una corriente obtenida de la aportación de aguas del subsuelo o de otras fuentes independientes. Sin embargo, resulta preocupante la contaminación de las aguas superficiales, ya que además de fenómenos naturales a las que están expuestas por ser superficiales, existe, la contaminación de 
manera directa causada por el hombre. El suministro de agua depende de los recursos de agua; el agua utilizada se devuelve al ciclo medioambiente-agua de un modo esperanzador después del tratamiento apropiado. De tal forma que el suministro de agua tiene que ser entendido como una parte del ciclo de agua y tiene que ser tratado dentro de la estructura de una gestión integral de recursos de agua. (Merkel, 2003)

Las principales fuentes de agua superficial que pueden explotarse incluyen, embalses o lagos, corrientes superficiales y canales para irrigación. Excepto en el caso de agua para irrigación cuyas descargas dependen de las actividades de riego, las fuentes mencionadas obtienen de precipitaciones directas sobre la zona de escurrimiento. La contaminación física y bacteriológica de las aguas superficiales, con la excepción de zonas de poca densidad de población, obliga a considerar tales fuentes de abastecimiento como inseguras para usos domésticos a menos que se proporcione procesos de potabilización confiables que incluyan filtración y desinfección en caso necesario. (Torres, P Cruz, 2009). Los defectos de las aguas superficiales son más aparentes que en las aguas subterráneas, de modo que se debe tratar de manera diferente cada una, de acuerdo con las características y propiedades de estas. Es importante saber la composición de cada una de las aguas para así determinar un tratamiento óptimo. (Guerrero, 1976). La selección y utilización de fuentes de agua superficial para sistemas particulares de abastecimiento de agua requieren que se tomen en consideración otros factores que no están, por lo general, asociados con las fuentes de agua del subsuelo. Como regla general las aguas superficiales deben utilizarse solamente en los casos en que no se disponga de fuentes de agua de subsuelo o que esta sea de calidad inadecuada. (Bogardi, 2003) El agua potable destinada al consumo humano debe cumplir ante todo con una calidad sanitaria apta, tanto inmediatamente después de su proceso de tratamiento, como presentar una estabilidad biológica en la red de distribución. (Romero, 2008)

El diseño de una planta de tratamiento requiere de cálculos de ingeniería con base en la caracterización físico-química del agua de la fuente y de la acertada selección de las operaciones de potabilización del agua, de tal manera que es necesario realizar los estudios de tratabilidad para determinar el tipo de planta requerida. (Lee \& Kim, 2007). El tratamiento de agua tiene por finalidad producir los cambios necesarios para acondicionarla a los patrones de calidad recomendados para el consumo humano y esto se logra a través de la instalación de plantas de tratamiento de aguas para entregar agua cuyas características físicas, químicas y microbiológicas estén enmarcadas dentro de las normas vigentes y además, entregarla en cantidad suficiente, con la continuidad requerida, para satisfacer las necesidades de la población.(Vidal, Martínez, \& Ayza, 1994)

Los desarenadores forman parte de una planta de potabilización de agua y son unidades que tienen por objeto extraer del agua cruda, la gravilla, arena y partículas minerales que tienen un tamaño superior a 200 micras, de tal manera que se evite la producción de sedimentos en los canales y conducciones. El diseño del desarenador se realiza en base al 
análisis de los fenómenos de sedimentación de partículas granuladas no floculantes las cuales sedimentan independientemente unas de otras, no existiendo interacción significativa entre las más próximas. El estudio de las velocidades de sedimentación se puede realizar utilizando las fórmulas de Stokes (régimen laminar), de Newton (régimen turbulento) de Allen (régimen transitorio). (Camacho, 2011)

Los medidores de caudales permiten conocer con precisión el caudal ingresado a la planta, los sistemas que podemos utilizar son varios, sin embargo, los usuales son sistemas basados en canales abiertos. Cuando la capacidad nominal de la medición de caudales en plantas sea menos a $1,0 \mathrm{~m} 3 / \mathrm{s}$ se podrán utilizar medidores tipo parshall, vertederos calibrados y otros dispositivos tales como los venturi. La estructura hidráulica de un canal parshall permite medir la cantidad de agua que pasa por una sección del canal. (Monroy, 2010)

En la operación de sedimentación mediante la fuerza de gravedad se remueven los sólidos en suspensión. En los sedimentadores clásicos estos sólidos en suspensión no deben ser de origen coloidal. Los diámetros están comprendidos entre 0,01 mm y 0,20 mm. Los sedimentadores son de sección rectangular compuestos por las siguientes zonas: entrada, sedimentación, lodos y salida. (Quiñones, Bustos, Vives, Miranda, \& Villarreal, 2014)

La filtración se considera como el paso de un fluido a través de un medio poroso que retiene los sólidos que se encuentra en suspensión. Se emplea para obtener una mayor clarificación, generalmente se aplica después de la sedimentación para eliminar las sustancias que no salieron del agua durante su decantación. En las principales instalaciones de filtración, los filtros sueles ser abiertos, mientras los filtros cerrados suelen utilizarse para instalaciones pequeñas (menor de 40m3/h). (Boccelli, Small, \& Diwekar, 2004) En las instalaciones de filtración de las estaciones de tratamiento de agua, el medio poroso suele ser generalmente arena, arena más antracita o bien carbón activo en grano, y la materia en suspensión está constituida por flóculos o microflóculos procedentes de la etapa anterior de decantación o bien formados expresamente cuando se sigue el proceso conocido como micro floculación sobre filtro o filtración directa. (Vandijk, J. C.; Oomen, 1978)

El proceso de desinfección es considerado de mayor importancia en la potabilización del agua. La desinfección es necesaria para la destrucción de todas las bacterias patógenas o de otros organismos dañinos que se encuentren en el agua para beber. Después de la desinfección para evitar que el agua se vuelva a contaminar, es necesario almacenarla en tanques adecuados o en instalaciones que reúnen buenas condiciones de almacenamiento. La cloración es el procedimiento de desinfección de aguas mediante el empleo de cloro o compuestos clorados. Se emplea gas cloro, pero normalmente se emplea hipoclorito de sodio por su mayor facilidad de almacenamiento y dosificación. (Bersillon, 1999). Sin embargo, el proceso tiene sus limitaciones, giardia lamblia y cryptosporidium son usualmente resistentes al cloro, a menos que este se use en dosis más elevadas que 
aquellas preferidas usualmente para el tratamiento. La presencia de estos parásitos puede requerir el tratamiento previo del agua fuente. (Rodríguez, Rodríguez, Serodes, \& Sadiq, 2007).

\section{Metodología.}

El método de investigación que se utiliza es de tipo comparativo que relacionado con los datos recogidos durante la investigación nos permitirán diseñar el sistema de tratamiento de agua potable. Para la caracterización del agua cruda se realiza un muestreo sistemático simple en la captación durante 3 días a la semana por 4 semanas consecutivas. Las condiciones meteorológicas en la primera semana presentan lluvias constantes, y las 3 semanas siguientes tuvieron un clima moderado sin lluvias. Se realiza análisis físico químicos del agua muestreada en el Laboratorio de Control de Calidad de Agua.

El análisis de los valores de caracterización obtenido del agua se realiza con base en la Norma Técnica INEN 1108:2014 referida a Requisitos de Agua Potable y las determinaciones física y químicas se realizan según los siguientes métodos, Tabla 1.

Tabla 1. Descripción de métodos de análisis

\begin{tabular}{|c|c|c|c|}
\hline Determinaciones & Parámetro & $\begin{array}{l}\text { Unidad de } \\
\text { medida }\end{array}$ & Métodos \\
\hline \multirow[t]{2}{*}{ Físicas } & $\begin{array}{c}\text { pH } \\
\text { Temperatura } \\
\text { Turbiedad } \\
\text { Conductividad } \\
\text { Sólidos Totales } \\
\text { Disueltos }\end{array}$ & $\begin{array}{c}{ }^{\circ} \mathrm{C} \\
\mathrm{NTU} \\
\mathrm{us} / \mathrm{cm} . \\
\mathrm{mg} / \mathrm{L}\end{array}$ & $\begin{array}{l}\text { Potenciométrico } \\
\text { Termómetro } \\
\text { Turbidímetro } \\
\text { Conductímetro } \\
\text { Conductímetro }\end{array}$ \\
\hline & & $\begin{array}{l}\mathrm{Pt}-\mathrm{Co} \\
\mathrm{m}^{3} / \mathrm{s} \\
\mathrm{s}\end{array}$ & $\begin{array}{l}\text { Fotómetro Hach } \\
\text { Volumétrico } \\
\text { Cronómetro }\end{array}$ \\
\hline \multirow{4}{*}{ Químicas } & $\begin{array}{c}\text { Hierro } \\
\text { Manganeso }\end{array}$ & $\begin{array}{l}\mathrm{mg} / \mathrm{L} \\
\mathrm{mg} / \mathrm{L}\end{array}$ & $\begin{array}{c}\text { Espectrofotómetro Hach } \\
\text { DR/2010 } \\
\text { Espectrofotómetro Hach } \\
\text { DR/2010 }\end{array}$ \\
\hline & $\begin{array}{l}\text { Amonio } \\
\text { Nitratos } \\
\text { Nitritos } \\
\text { Fosfatos }\end{array}$ & $\begin{array}{l}\mathrm{mg} / \mathrm{L} \\
\mathrm{mg} / \mathrm{L} \\
\mathrm{mg} / \mathrm{L} \\
\mathrm{mg} / \mathrm{L}\end{array}$ & $\begin{array}{c}\text { Espectrofotómetro Hach } \\
\text { DR/2010 } \\
\text { Espectrofotómetro Hach } \\
\text { DR/2010 } \\
\text { Espectrofotómetro Hach } \\
\text { DR/2010 }\end{array}$ \\
\hline & Calcio & $\mathrm{mg} / \mathrm{L}$ & $\begin{array}{c}\text { Espectrofotómetro Hach } \\
\text { DR/2010 }\end{array}$ \\
\hline & Alcalinidad Total & $\mathrm{mg} / \mathrm{L}$ & $\begin{array}{c}\text { Volumétricos y/o } \\
\text { Espectrofotómetro Hach } \\
\text { DR/2010 }\end{array}$ \\
\hline
\end{tabular}




\begin{tabular}{|c|c|c|c|}
\hline & Dureza Total & $\mathrm{mg} / \mathrm{L}$ & $\begin{array}{c}\text { Volumétricos y/o } \\
\text { Espectrofotómetro Hach } \\
\text { DR/2010 } \\
\text { Volumétricos y/o } \\
\text { Espectrofotómetro Hach } \\
\text { DR/2010 }\end{array}$ \\
\hline Microbiológicas & $\begin{array}{l}\text { Coliformes } \\
\text { Totales } \\
\text { Coliformes } \\
\text { Fecales }\end{array}$ & $\begin{array}{l}\mathrm{UFC} / 100 \mathrm{ml} \\
\mathrm{UFC} / 100 \mathrm{ml}\end{array}$ & $\begin{array}{l}\text { Método por Filtro de } \\
\text { Membrana } \\
\text { Método por Filtro de } \\
\text { Membrana }\end{array}$ \\
\hline
\end{tabular}

Fuente: Laboratorio Control de Calidad de Agua

Con base en las caracterizaciones físicas - químicas y microbiológicas del agua se determina el tipo de tratamiento necesario de tal manera que subsanen los parámetros que no cumplan con la Norma Técnica INEN 1108:2014.

Los equipos que pertenecen a las etapas de potabilización de agua para su construcción requieren de cálculos de ingeniería según la información obtenida en la caracterización de agua cruda, para el dimensionamiento se utilizan las siguientes ecuaciones:

Tabla 2. Ecuaciones diseño de medidor parshall

\begin{tabular}{|c|c|c|c|}
\hline Cálculo & Símbolo & Ecuación & \\
\hline Sumergencia máxima & S & $\mathrm{S}=\frac{\mathrm{H}_{\mathrm{b}}}{\mathrm{H}_{\mathrm{a}}}$ & Ec. 1 \\
\hline \multirow{2}{*}{ Altura de la cresta } & \multirow{2}{*}{$\mathrm{Ha}$} & $\mathrm{Q}^{\frac{1}{1,57 * \mathrm{w}^{0,026}}}$ & \multirow{2}{*}{ Ec. 2} \\
\hline & & $(0,3716 \mathrm{~W})^{\frac{1}{1,57 \mathrm{w}^{0,026}} * 3,281}$ & \\
\hline $\begin{array}{l}\text { Altura de agua sobre la } \\
\text { garganta }\end{array}$ & $\mathrm{Hb}$ & $S=\frac{H b}{H a}$ & Ec. 3 \\
\hline Pérdida de carga & $\mathrm{P}$ & $P=\frac{5,072}{(W+4,57)^{1,46}}(1-S)^{0,72} * Q^{0,67}$ & Ec. 4 \\
\hline
\end{tabular}

Fuente: (Arboleda, 2000)

Tabla 3. Ecuaciones de diseño de sedimentador

\begin{tabular}{lccc}
\hline \multicolumn{1}{c}{ Cálculo } & Símbolo & Ecuación & \\
\hline Velocidad de la sedimentación & $\mathrm{V}_{\mathrm{S}}$ & $\mathrm{V}_{\mathrm{S}}=\frac{1}{18} \mathrm{~g}\left\langle\frac{\rho_{\mathrm{s}}-\rho_{\mathrm{H}_{2} \mathrm{O}}}{\mathrm{n}}\right\rangle \mathrm{d}^{2}$ & Ec. $\mathbf{5}$ \\
Velocidad de Sedimentación crítica & $\mathrm{V}_{\mathrm{SC}}$ & $\mathrm{V}_{\mathrm{sc}}=\frac{\mathrm{V}_{\mathrm{s}}}{\mathrm{f}}$ & $\mathrm{A}=\frac{\mathrm{v}}{\mathrm{H}} \mathbf{6}$ \\
Altura del sedimentador & $\mathrm{H}$ & $\mathrm{L}=4 * \mathrm{~B}$ & Ec. 7 \\
Longitud del sedimentador & $\mathrm{L}$ & $\mathrm{B}=\sqrt{\frac{\mathrm{A}_{\mathrm{i}}}{4}} \mathbf{8}$ & Ec. $\mathbf{9}$
\end{tabular}


Velocidad de escurrimiento

Velocidad de arrastre de las partículas

Volumen diario de lodos

Velocidad de paso de agua por deflector

Número de orificios para deflector

Diámetro de cada orificio de deflector

Caudal de cada vertedero

$\begin{array}{ccc}V_{h} & V_{h}=\frac{Q}{B * H} & \text { Ec. } 10 \\ V_{a} & V_{a}=\sqrt{40 *\left(\rho_{s}-\rho_{w}\right) * g * d /\left(3 * \rho_{w}\right)} & \text { Ec. 11 } \\ V_{1-24 h} & V_{1}=E * C * Q & \text { Ec. 12 } \\ V_{C} & V_{C}=\frac{Q}{n * d * 0,9 * H} & \text { Ec. 13 } \\ & \frac{B}{0,3} * \frac{H}{0,3} & \text { Ec. 14 } \\ \mathrm{F}_{\mathrm{w}} & \mathrm{F}_{\mathrm{i}}=\Pi * \mathrm{r}^{2} & \text { Ec. 15 } \\ \mathrm{N}_{\mathrm{c}} * \mathrm{~N}_{\mathrm{w}} & \text { Ec. 16 }\end{array}$

Fuente: (Basile, 2018)

Tabla 4. Ecuaciones de diseño de filtros

\begin{tabular}{cccc}
\hline Cálculo & Símbolo & Ecuación & \\
\hline Número de filtros totales & $\mathrm{N}$ & $\mathrm{n}=0,5 * \sqrt[3]{\mathrm{A}}$ & Ec. 17 \\
Longitud & $\mathrm{L}$ & $\mathrm{a}=\left(\frac{2 * \mathrm{n} * \mathrm{~A}_{\mathrm{i}}}{2 * 1}\right)^{0,5}$ & Ec. 18 \\
Ancho & $\mathrm{B}$ & $\mathrm{b}=\left(\frac{(n+1) A_{i}}{2 * \mathrm{n}}\right)^{0,5}$ & Ec. 19 \\
\hline
\end{tabular}

Fuente: (Barraque, 1979)

\section{Resultados.}

Los resultados del análisis físico del agua según Tabla 5 manifiestan que durante la primera semana el parámetro de turbiedad no se encuentra dentro de los límites permisible bajo la Norma Técnica INEN 1108:2014, durante la recolección de estas muestras se evidenció arrastre de material fino por las tuberías de captación debido a la presencia de lluvias moderadas. Los resultados de los análisis de agua tratada en las siguientes semanas se encuentran dentro de los límites permisibles.

Tabla 5. Caracterización física del agua cruda

\begin{tabular}{ccccccc}
\hline Muestra & pH & $\begin{array}{c}\text { Color } \\
\text { NTU }\end{array}$ & $\begin{array}{c}\text { Turbiedad } \\
\text { NTU }\end{array}$ & $\begin{array}{c}\text { Conductividad } \\
\mathbf{u S} / \mathbf{c m}\end{array}$ & $\begin{array}{c}\text { STD } \\
\mathbf{m g} / \mathbf{L}\end{array}$ & $\begin{array}{c}\text { Temperatura } \\
\mathbf{C}\end{array}$ \\
\hline $\begin{array}{c}\text { Límites } \\
\text { permisibles }\end{array}$ & $6,5-8,5$ & 15 & 5 & & 1000 & \\
1 & 7,49 & 5 & 5,6 & 728 & 355 & 20,1 \\
2 & 7,81 & 5 & 6,4 & 803 & 406 & 17
\end{tabular}




\begin{tabular}{ccccccc}
\hline 3 & 7,62 & 5 & 5,2 & 679 & 350 & 17,4 \\
4 & 7,78 & 5 & 1,8 & 113,7 & 54 & 16,2 \\
5 & 7,83 & 5 & 1,5 & 110,6 & 52,6 & 17,5 \\
6 & 7,8 & 5 & 1,6 & 111,4 & 53,2 & 17,1 \\
7 & 7,62 & 5 & 2 & 124,4 & 59,2 & 18,7 \\
8 & 7,82 & 5 & 1,6 & 123,8 & 58,9 & 16,9 \\
9 & 7,63 & 5 & 1,5 & 124,2 & 59 & 18 \\
10 & 6,64 & 5 & 1,6 & 128,8 & 61,3 & 18,6 \\
11 & 7,64 & 5 & 1,7 & 129,4 & 61,6 & 17,5 \\
12 & 7,47 & 5 & 1,6 & 127,5 & 60,7 & 18,3 \\
\hline
\end{tabular}

Fuente: Elaboración propia, (INEN, 2014)

Los resultados de los análisis químicos de fosfatos según Tabla 6 no se encuentran dentro del límite permisible según la Norma Técnica INEN 1108. El incremento de la concentración de fosfatos causa la eutrofización del agua pues la presencia de fósforo genera el crecimiento de algas. (Correl, 1998)

Tabla 6. Caracterización química del agua cruda

\begin{tabular}{|c|c|c|c|c|c|c|c|}
\hline Muestra & $\begin{array}{c}\text { Fosfatos } \\
\text { mg/L }\end{array}$ & $\begin{array}{c}\text { Hierro } \\
\mathrm{mg} / \mathrm{L}\end{array}$ & $\begin{array}{c}\text { Manganeso } \\
\mathrm{mg} / \mathrm{L}\end{array}$ & $\begin{array}{c}\text { Nitritos } \\
\mathrm{mg} / \mathrm{L}\end{array}$ & $\begin{array}{c}\text { Nitratos } \\
\mathrm{mg} / \mathrm{L}\end{array}$ & $\begin{array}{c}\mathrm{N}- \\
\text { Amoniacal } \\
\mathrm{mg} / \mathrm{L}\end{array}$ & $\begin{array}{c}\text { Sulfatos } \\
\mathrm{mg} / \mathrm{L}\end{array}$ \\
\hline $\begin{array}{c}\text { Límites } \\
\text { permisibles }\end{array}$ & 0,1 & 0,3 & 0,1 & 0 & 10 & 1 & 200 \\
\hline 1 & 0,13 & 0,2 & 0,001 & 0,003 & 0 & 0,004 & 8 \\
\hline 2 & 0,1 & 0,01 & 0,001 & 0,001 & 0 & 0,004 & 8 \\
\hline 3 & 0,12 & 0,01 & 0,001 & 0 & 0 & 0,03 & 8 \\
\hline 4 & 0,34 & 0,08 & 0,001 & 0 & 0 & 0,04 & 8 \\
\hline 5 & 0,27 & 0,04 & 0,003 & 0 & 0 & 0,02 & 8 \\
\hline 6 & 0,31 & 0,05 & 0,001 & 0 & 0,001 & 0,02 & 8 \\
\hline 7 & 0,6 & 0,02 & 0,05 & 0,04 & 0 & 0,03 & 8 \\
\hline 8 & 0,03 & 0,02 & 0 & 0,003 & 0 & 0,05 & 7 \\
\hline 9 & 0,38 & 0,01 & 0,001 & 0 & 0,1 & 0,05 & 8 \\
\hline 10 & 0,81 & 0 & 0 & 0 & 0,3 & 0,05 & 7 \\
\hline 11 & 0,64 & 0 & 0,001 & 0 & 0,3 & 0 & 8 \\
\hline 12 & 0,43 & 0,04 & 0,001 & 0 & 0 & 0 & 8 \\
\hline
\end{tabular}

Fuente: Elaboración propia, (INEN, 2014)

Los análisis microbiológicos del agua cruda según Tabla 7 están fuera de los límites permisibles bajo la Norma Técnica INEN 1108. 
Tabla 7. Caracterización microbiológica del agua cruda

\begin{tabular}{ccc}
\hline Muestras & $\begin{array}{c}\text { CT } \\
\text { UFC/100ml }\end{array}$ & $\begin{array}{c}\text { CF } \\
\text { UFC/100ml }\end{array}$ \\
\hline Límite Permisible & $<1$ & $<1$ \\
1 & 222 & 98 \\
2 & 298 & 96 \\
3 & 196 & 71 \\
4 & 132 & 28 \\
5 & 249 & 125 \\
6 & 116 & 12 \\
7 & 275 & 28 \\
8 & 70 & 2 \\
9 & 365 & 6 \\
10 & 454 & 58 \\
11 & 365 & 9 \\
12 & 248 & 204 \\
\hline
\end{tabular}

Fuente: Elaboración propia, (INEN, 2014)

Figura. 1 Parámetros fuera de Norma INEN 1108:2014

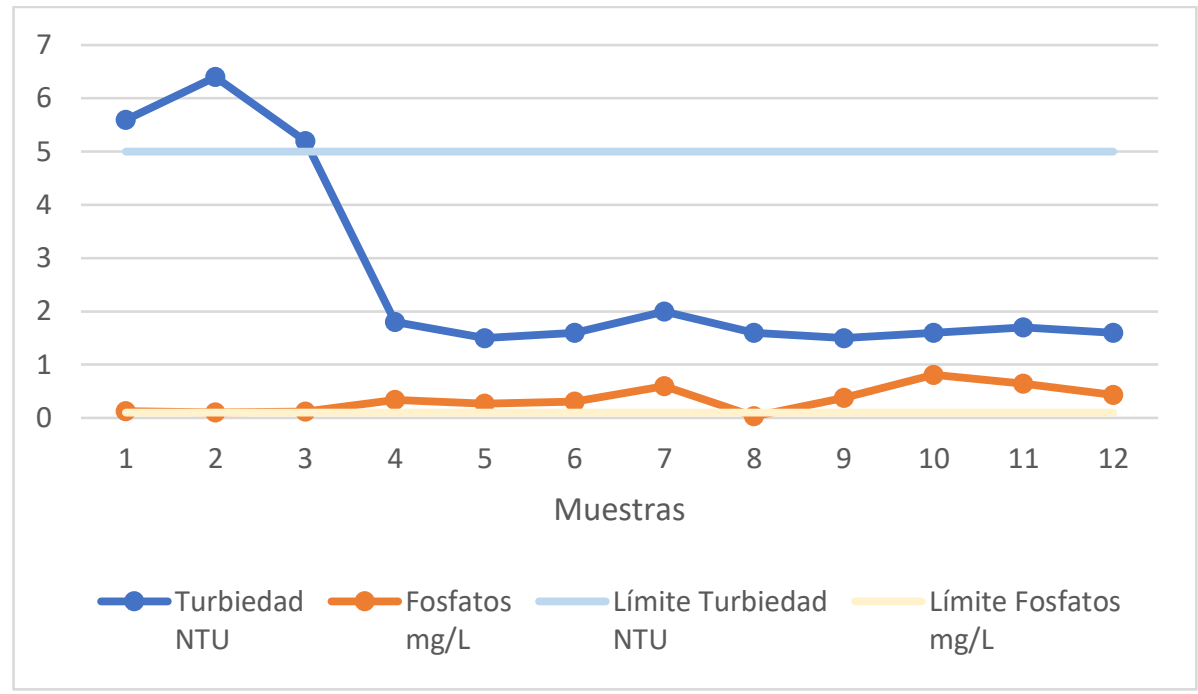

Fuente: Elaboración propia

Se realizan pruebas para determinar el $\mathrm{pH}$, conductividad y alcalinidad de grava y arena fina según Tabla 8 y 9, materiales que serán aplicados en los filtros de la planta de tratamiento. 
Tabla 8. Determinación de parámetros de la grava seca de filtro grueso ascendente

\begin{tabular}{cccc}
\hline Muestras & $\mathbf{p H}$ & Conductividad & Alcalinidad \\
\hline $\mathbf{1}$ & 6,8 & 56,3 & 28 \\
$\mathbf{2}$ & 7,75 & 25,4 & 23 \\
$\mathbf{3}$ & 7,9 & 32,6 & 21 \\
\hline
\end{tabular}

Fuente: Elaboración propia

Tabla 9. Determinación de parámetros de la arena seca del filtro lento

\begin{tabular}{cccc}
\hline Muestras & $\mathbf{p H}$ & Conductividad & Alcalinidad \\
\hline $\mathbf{1}$ & 6,4 & 59,7 & 28 \\
$\mathbf{2}$ & 6,7 & 22,6 & 19 \\
$\mathbf{3}$ & 6,8 & 19,3 & 17 \\
\hline
\end{tabular}

Fuente: Elaboración propia

Se realizan pruebas de sedimentación y filtración en el laboratorio, utilizando grava y arena fina lavadas, secadas y tamizadas. En estas pruebas el parámetro que se mide es la turbiedad (NTU) debido a que los datos recolectados se encuentran fuera de los límites permisibles bajo la Norma Técnica INEN 110:2014 y que ocasionarían dificultades en el tratamiento del agua, según se aprecia en Tabla 10.

Tabla 10. Pruebas de Tratabilidad prueba turbiedad

\begin{tabular}{ccccc}
\hline Prueba & $\begin{array}{c}\text { Muestra } \\
\text { (Agua Cruda) }\end{array}$ & $\begin{array}{c}\text { Volumen } \\
\text { L }\end{array}$ & $\begin{array}{c}\text { Turbiedad } \\
\text { (Agua Cruda) } \\
\text { NTU }\end{array}$ & $\begin{array}{c}\text { Turbiedad } \\
\text { (Agua Tratada) } \\
\text { NTU }\end{array}$ \\
\hline 1 & 1 & 8 & 5,6 & 0,41 \\
2 & 1 & 8 & 6.8 & 0,5 \\
3 & 1 & 8 & 4,3 & 0,31 \\
4 & 1 & 8 & 5,2 & 0,38 \\
5 & 1 & 8 & 6,1 & 0,45 \\
\hline
\end{tabular}

Fuente: Elaboración propia

En el laboratorio se desarrolla pruebas de tratabilidad para reducir la concentración de fosfatos en el agua cruda que según los resultados de la caracterización química se encuentran fuera del límite permisible según normativa vigente. Para estas pruebas se 
añade dosificaciones de solución de sulfato de cobre de 3, 4 y $5 \mathrm{~mL}$ en un litro de agua cruda.

Tabla 11. Pruebas de tratabilidad parámetro fosfatos para $1 \mathrm{~L}$ de agua cruda

\begin{tabular}{cccc}
\hline Prueba & $\begin{array}{c}\text { Fosfatos } \\
\text { (Agua Cruda) } \\
\mathbf{m g} / \mathbf{L}\end{array}$ & $\begin{array}{c}\text { Solución de } \\
\text { Sulfato de Cobre } \\
\mathbf{( 3 m L / s )}\end{array}$ & $\begin{array}{c}\text { Fosfatos } \\
\text { (Agua Tratada) } \\
\mathbf{m g} / \mathbf{L}\end{array}$ \\
\hline 1 & 0,18 & 3 & 0,12 \\
2 & 0,23 & 3 & 0,14 \\
3 & 0,12 & 3 & 0,11 \\
4 & 0,14 & 3 & 0,11 \\
5 & 0,25 & 3 & 0,15 \\
\hline
\end{tabular}

Fuente: Elaboración propia

Tabla 12. Pruebas de tratabilidad parámetro fosfatos $1 \mathrm{~L}$ de agua cruda

\begin{tabular}{cccc}
\hline Prueba & $\begin{array}{c}\text { Fosfatos } \\
\text { (Agua Cruda) } \\
\mathbf{m g} / \mathbf{L}\end{array}$ & $\begin{array}{c}\text { Solución de } \\
\text { Sulfato de Cobre } \\
\mathbf{( 4 ~} \mathbf{~} \mathbf{L} / \mathbf{s})\end{array}$ & $\begin{array}{c}\text { Fosfatos } \\
\text { (Agua Tratada) } \\
\mathbf{m g} / \mathbf{L}\end{array}$ \\
\hline 1 & 0,18 & 4 & 0,08 \\
2 & 0,23 & 4 & 0,09 \\
3 & 0,12 & 4 & 0,08 \\
4 & 0,14 & 4 & 0,07 \\
5 & 0,25 & 4 & 0,09 \\
\hline
\end{tabular}

Fuente: Elaboración propia

Tabla 13. Pruebas de tratabilidad parámetro fosfatos $1 \mathrm{~L}$ de agua cruda

\begin{tabular}{cccc}
\hline Prueba & $\begin{array}{c}\text { Fosfatos } \\
\text { (Agua Cruda) } \\
\mathbf{m g} / \mathbf{L}\end{array}$ & $\begin{array}{c}\text { Solución de } \\
\text { Sulfato de Cobre } \\
\mathbf{5} \mathbf{~ m L / s})\end{array}$ & $\begin{array}{c}\text { Fosfatos } \\
\text { (Agua Tratada) } \\
\mathbf{~ m g / L}\end{array}$ \\
\hline 1 & 0,18 & 5 & 0,032 \\
2 & 0,23 & 5 & 0,037 \\
3 & 0,12 & 5 & 0,024 \\
4 & 0,14 & 5 & 0,027 \\
5 & 0,25 & 5 & 0,026 \\
\hline
\end{tabular}

Fuente: Elaboración propia

El porcentaje de remoción de fosfatos con las dosis de 3 y $4 \mathrm{~mL}$ según las tablas 11 y 12 resultan en valores fuera de los límites permisibles para fosfatos bajo la Norma Técnica INEN 1108:2014. Sin embargo, la dosificación de $5 \mathrm{~mL}$ de sulfato de cobre logra la disminución de 82,5\% promedio de la concentración de fosfatos en las muestras probadas según Fig. 2, permitiendo cumplir con el parámetro establecido según normativa. 
Tabla 14. Resultados de los porcentajes de remoción en los fosfatos con $5 \mathrm{~mL}$ de solución de sulfato de cobre

\begin{tabular}{ccccc}
\hline Muestra & $\begin{array}{c}\text { Límite } \\
\text { Permisible } \\
(\mathbf{m g} / \mathbf{L})\end{array}$ & $\begin{array}{c}\text { Fosfatos Agua Cruda } \\
(\mathbf{m g} / \mathbf{L})\end{array}$ & $\begin{array}{c}\text { Fosfatos Agua } \\
\text { Tratada }(\mathbf{m g} / \mathbf{L})\end{array}$ & $\begin{array}{c}\text { Remoción } \\
\text { lograda } \\
\mathbf{\%}\end{array}$ \\
\hline 1 & 0,1 & 0,18 & 0,032 & 82,22 \\
2 & 0,1 & 0,23 & 0,037 & 83,91 \\
3 & 0,1 & 0,12 & 0,024 & 80,00 \\
4 & 0,1 & 0,14 & 0,027 & 80,71 \\
5 & 0,1 & 0,25 & 0,036 & 85,60 \\
\hline
\end{tabular}

Fuente: Elaboración Propia

Figura 2. Porcentaje de remoción de fosfatos en un litro de agua cruda con $5 \mathrm{~mL}$ de sulfato de cobre

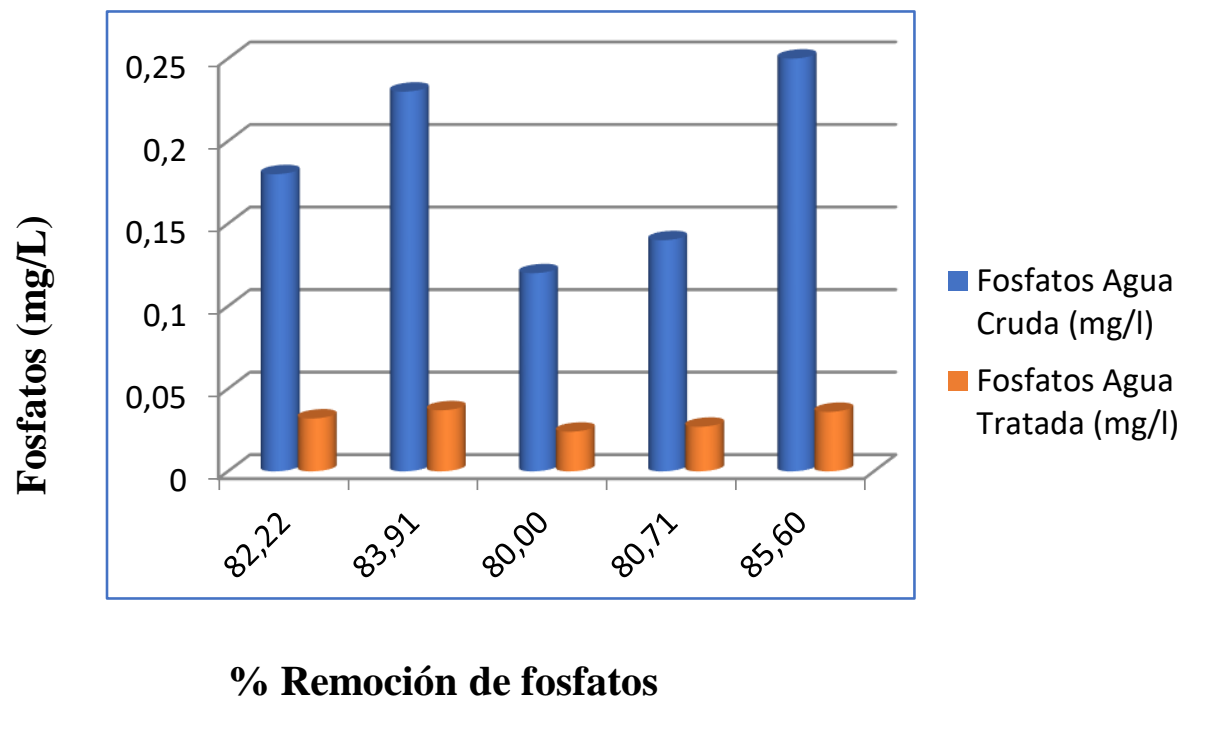

Fuente: Elaboración propia

Con un caudal promedio de $24 \mathrm{~L} / \mathrm{s}$ de captación de agua se realiza el diseño de la planta de tratamiento de agua con base en los resultados obtenidos en la caracterización y las ecuaciones planteadas para un medidor de caudal parshall, sedimentadores, filtros gruesos ascendentes y filtros lentos según las Tablas de diseño presentadas a continuación. 
Tabla 15. Diseño del Medidor Parshall

\begin{tabular}{lccc}
\hline \multicolumn{1}{c}{ Cálculos } & Símbolo & Valor & Unidades \\
\hline Sumergencia máxima & $\mathrm{S}$ & 0,6 & $\mathrm{~m} / \mathrm{m}$ \\
Ancho de la garganta & $\mathrm{W}$ & 0,229 & $\mathrm{~m}$ \\
Altura de la cresta & $\mathrm{Ha}$ & 0,132 & $\mathrm{~m}$ \\
Altura de agua sobre la garganta & $\mathrm{Hb}$ & 0,079 & $\mathrm{~m}$ \\
Pérdida de carga & $\mathrm{P}$ & 0,22 & $\mathrm{~m}$ \\
Dimensiones estandarizadas de & & & \\
secciones de canal de acuerdo con & & Para W $=0,229$ & \\
Anexo H & & & \\
\hline
\end{tabular}

Fuente: Elaboración propia

Tabla 16. Diseño de sedimentadores clásicos

\begin{tabular}{lccc}
\hline \multicolumn{1}{c}{ Cálculos } & Símbolo & Valor & Unidades \\
\hline Número de sedimentadores & $\mathrm{N}$ & 2 & \\
Volumen del sedimentador & $\mathrm{V}$ & 216 & $\mathrm{~m}^{3}$ \\
Tiempo de sedimentación & $\mathrm{T}$ & 2,5 & $\mathrm{H}$ \\
Velocidad de sedimentación & $\mathrm{V}_{\mathrm{S}}$ & 0,34 & $\mathrm{~mm} / \mathrm{s}$ \\
Velocidad de Sedimentación Crítica & $\mathrm{V}_{\mathrm{SC}}$ & 0,26 & $\mathrm{~mm} / \mathrm{s}$ \\
Altura del sedimentador & $\mathrm{H}$ & 2,34 & $\mathrm{~m}$ \\
Longitud del sedimentador & $\mathrm{L}$ & 13,6 & $\mathrm{~m}$ \\
Ancho del sedimentador & $\mathrm{B}$ & 3.4 & $\mathrm{~m}$ \\
Velocidad de escurrimiento & $\mathrm{V}_{\mathrm{h}}$ & $3,017^{*} 10^{-3}$ & $\mathrm{~m} / \mathrm{s}$ \\
Velocidad de arrastre de las partículas & $\mathrm{V}_{\mathrm{a}}$ & 1,58 & $\mathrm{~m} / \mathrm{s}$ \\
Volumen diario de lodos & $\mathrm{V}_{1-24 \mathrm{~h}}$ & 32.83 & $\mathrm{~m}^{3} / \mathrm{s}$ \\
Altura de pantalla deflectora & $\mathrm{H}_{\mathrm{f}}$ & 2,11 & $\mathrm{~m}$ \\
Velocidad de paso de agua por deflector & $\mathbf{V}_{\mathrm{C}}$ & $5,69 * 10^{-3}$ & $\mathrm{~m} / \mathrm{s}$ \\
Número de orificios para deflector & & 77 & \\
Diámetro de cada orificio de deflector & $\Phi$ & 0,05 & $\mathrm{~m}$ \\
Número de Canaletas de salida & & 2 & \\
Longitud de canaleta & & 2 & $\mathrm{~m}$ \\
Número de vertederos por canaleta & $\mathrm{N}_{\mathrm{w}}$ & 14 & \\
Caudal de cada vertedero & $\mathrm{Q}_{\mathrm{w}}$ & $4,28 * 10^{-4}$ & $\mathrm{~m} 3 / \mathrm{s}$ \\
\hline
\end{tabular}

Fuente: Elaboración propia

Tabla 17. Diseño de filtros gruesos ascendentes

\begin{tabular}{lccc}
\hline \multicolumn{1}{c}{ Cálculos } & Símbolo & Valor & Unidades \\
\hline Número de filtros totales & $\mathrm{N}$ & 2 & \\
Velocidad de filtración & $\mathrm{V}_{\mathrm{f}}$ & 0,6 & $\mathrm{~m} / \mathrm{h}$ \\
Longitud y Base & $\mathrm{L}, \mathrm{b}$ & 8,49 & $\mathrm{~m}$ \\
Altura & $\mathrm{H}$ & 1,2 & $\mathrm{~m}$ \\
\hline
\end{tabular}

Fuente: Elaboración propia 
Tabla 18. Lecho para filtros gruesos ascendente

\begin{tabular}{cc}
\hline Grava $(\mathbf{m m})$ & Altura $(\mathbf{m})$ \\
\hline $19-25$ & 0,2 \\
$13-19$ & 0,2 \\
$6-13$ & 0,2 \\
$3-6$ & 0,2 \\
Soporte & 0,1 \\
\hline
\end{tabular}

Fuente: (Galvis G, Latorre J, 1998)

Tabla 19. Diseño de filtros lentos

\begin{tabular}{lccc}
\hline \multicolumn{1}{c}{ Cálculos } & Símbolo & Valor & Unidades \\
\hline Número de filtros totales & $\mathrm{N}$ & 3 & \\
Velocidad de filtración & $\mathrm{V}_{\mathrm{f}}$ & 0,3 & $\mathrm{~m} / \mathrm{h}$ \\
Longitud & $\mathrm{L}$ & 16,9 & $\mathrm{~m}$ \\
Ancho & $\mathrm{B}$ & 6,92 & $\mathrm{~m}$ \\
Altura total & $\mathrm{H}$ & 1,90 & $\mathrm{~m}$ \\
\hline
\end{tabular}

Fuente: Elaboración propia

Tabla 20. Lecho para filtros lentos de arena

\begin{tabular}{ccc}
\hline Lecho & $\begin{array}{c}\text { Diámetro } \\
(\mathbf{m m})\end{array}$ & $\begin{array}{c}\text { Altura } \\
(\mathbf{m})\end{array}$ \\
\hline Grava Gruesa & 1,2 & 0,3 \\
Arena de filtro & 0,22 & 0,9 \\
\hline
\end{tabular}

Fuente: (Galvis G, Latorre J, 1998)

\section{Conclusiones:}

- Los análisis físico-químicos del agua captada presentan especificaciones fuera de los límites permisibles bajo la Norma Técnica INEN 1108:2014 referidos a Requisitos de Agua Potable, para los parámetros de turbiedad de 5 NTU y para fosfatos $0,1 \mathrm{mg} / \mathrm{L}$, valores que fluctúan de acuerdo a las condiciones meteorológicas especialmente en invierno ocasionando obstrucción de filtros, de manera que se requiere de la etapa de sedimentación como tratamiento previo. La presencia de concentraciones altas de fosfatos en el agua captada genera el crecimiento de flora dependientes del fósforo como las algas, y la disminución de la calidad de agua para el consumo humano. La adición de 5 ML de sulfato de cobre por cada litro de agua cruda disminuye en un 82,5\% la concentración de fosfatos denotándola como apta para el consumo humano.

- La grava y la arena fina utilizados presentan índices de alcalinidad elevados que no destruyen completamente la población de coliformes termoresistentes, sin 
embargo, este escenario mejora generalmente con la adición de hipoclorito de sodio en la última etapa de tratamiento y según Norma Técnica INEN 1108:2014 deberá presentar un índice de cloro residual entre 0,3 y 1,5.

- A partir de un caudal máximo de tratamiento de $24 \mathrm{~L} / \mathrm{s}$ de captación de agua superficial se realizan cálculos de ingeniería y diseño para la planta de tratamiento de agua potable que contiene un medidor de caudales, dos sedimentadores, dos filtros ascendentes gruesos y tres filtros lentos con arena fina. El medidor de caudales es tipo parshall con un ancho de garganta de $\mathrm{W}=0,229$; el sedimentador clásico con una velocidad de sedimentación crítica $\mathrm{Vsc}=0,26 \mathrm{~mm} / \mathrm{s}$; el filtro ascendente grueso con una velocidad de filtración de $\mathrm{Vf}=0,6 \mathrm{~m} / \mathrm{h}$ y el filtro lento con una velocidad de filtración de $\mathrm{Vf}=0,3 \mathrm{~m} / \mathrm{h}$.

\section{Referencias bibliográficas:}

Arboleda, J. (2000). Teoría y Práctica de Purificación del Agua (3ra ed). Bogotá.

Barraque, C. (1979). Manual técnico del agua.

Basile, P. A. (2018). Transporte de sedimentos y morfodinamica de rios aluviales. In Biomass Chem Eng.

Bersillon, J.-L. (1999). Water Purification and Disinfection Processes. Acta Hydrochimica et Hydrobiologica, 27(2), 98-100.

Boccelli, D. L., Small, M. J., \& Diwekar, U. M. (2004). Treatment plant design for particulate removal: Effects of flow rate and particle characteristics. Journal / American Water Works Association, 96(11), 77-90.

Bogardi, J. J. (2003). Las políticas del agua en el siglo XXI. Science, 259-279.

Camacho, N. C. C. (2011). Tratamiento de agua para consumo humano. Ingeniería Industrial, 29, 153-224.

Correl, D. (1998). The Role of Phosphorus in the Eutrophication of Receiving Waters: A Review. Journal Environmental Quality.

Galvis G, Latorre J, T. J. (1998). Filtración en múltiples etapas: tecnología innovativa para el tratamiento de agua.

Guerrero, R. (1976). Manual de Tratamiento de Aguas. México: Limusa.

INEN. Norma Técnica Ecuatoriana. Agua Potable. Requisitos. , (2014).

Lee, \& Kim. (2007). Water-Treatment - System design for turbidity removal. Environmental Engineering, 67(6), 14-21.

Merkel, W. (2003). El futuro de la industria de agua en el mundo. Ingeniería Del Agua, $10(3), 337$.

Monroy, M. (2010). Medidores De Flujo En Canales Abiertos. Biblioteca.Usac.Edu.Gt, $1-119$.

Quiñones, E., Bustos, C., Vives, L., Miranda, V., \& Villarreal, A. (2014). Diseño de un sedimentador mediante la dinámica de fluidos computacional y su construcción a escala de laboratorio. (4), 161-167. 
Rodríguez, M. J., Rodríguez, G., Serodes, J., \& Sadiq, R. (2007). Subproductos de la desinfección del agua potable: Formación, aspectos sanitarios y reglamentación. Interciencia, 32(11), 749-756.

Romero, M. (2008). Tratamientos utilizados en La potabilización de agua. Boletin Electronico Facultad de Ingeniería - Universidad Rafael Landívar, (08), 1-12.

Torres, P Cruz, C. (2009). Índices De Calidad De Agua En Fuentes Superficiales Utilizadas En La Producción De Agua Para Water Quality Index in Surface Sources Used in Water Production for Human Consumption . a Critical Review. 8(15), 79-94.

Vandijk, J. C.; Oomen, J. H. (1978). Filtración lenta en arena para abastecimiento público de agua en países en desarrollo: manual de diseño y construcción. La Haya. CIROMS.

Vidal, R., Martínez, F., \& Ayza, M. (1994). Aplicaciones de los modelos de calidad en la simulación de las redes de distribución de agua potable. Ingeniería Del Agua, 1(3), $55-68$. 


\section{PARA CITAR EL ARTÍCULO INDEXADO.}

Flores Huilcapi, A. G., Carrera Almendáriz, L. S., \& Medina Serrano , C. A. (2020). Análisis de aguas superficiales con alto contenido de fosfatos para el diseño de una planta de tratamiento de agua potable. ConcienciaDigital,3(3), 27-43. https://doi.org/10.33262/concienciadigital.v3i3.1264

\section{\Ciencia}

El artículo que se publica es de exclusiva responsabilidad de los autores y no necesariamente reflejan el pensamiento de la Revista Conciencia Digital.

El artículo queda en propiedad de la revista y, por tanto, su publicación parcial y/o total en otro medio tiene que ser autorizado por el director de la Revista Conciencia Digital.

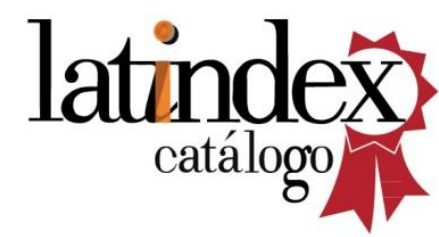

